\title{
OBSERVATIONS OF COMET 1889 V, 1896 VI (BROOKS)
}

\begin{tabular}{|c|c|c|c|c|c|c|c|c|c|c|c|c|c|c|c|}
\hline \multirow{4}{*}{$\begin{array}{c}1903 . \\
\text { Dec. } \\
" "\end{array}$} & \multirow{2}{*}{\multicolumn{4}{|c|}{ Mt. Hamilton M.T. }} & \multirow{3}{*}{$\begin{array}{c}\text { Star } \\
2\end{array}$} & \multirow{2}{*}{$\begin{array}{c}\text { No. of } \\
\text { Comparisons }\end{array}$} & \multicolumn{3}{|c|}{ Comet-Star } & \multicolumn{4}{|c|}{ Comet's Apparent } & \multicolumn{2}{|c|}{$\log p \Delta$} \\
\hline & & & & & & & $\Delta a$ & $\Delta$ & & & $a$ & & $\delta$ & $a$ & $\delta$ \\
\hline & 10 & $7^{\mathrm{h}}$ & $17^{\mathrm{m}}$ & $49^{\mathrm{s}}$ & & 10,6 & -1844 & $-0^{\prime}$ & $15 " .6$ & $22^{\mathrm{b}}$ & $27^{\mathrm{m}}$ & 38.52 & $-11^{\circ} 18^{\prime} 15^{\prime \prime} 7$ & 9.392 & 0.805 \\
\hline & 20 & 7 & 55 & 26 & 3 & 10,6 & +13.44 & -5 & 07.3 & 22 & 46 & 27.23 & -85343.1 & 9.531 & 0.778 \\
\hline an. & 7 & 7 & 18 & 12 & 5 & 10 & -6.05 & +0 & 47.6 & 23 & 21 & 37.79 & -42234.7 & 9.523 & 0.753 \\
\hline$"$ & 14 & 7 & 05 & 59 & 7 & 12,6 & +3.50 & +0 & 35.6 & 23 & 35 & 37.36 & -23434.7 & 9.525 & 0.742 \\
\hline
\end{tabular}

Mean Places of Comparison Stars for 1903.0 and 1904.0

\begin{tabular}{|c|c|c|c|c|c|c|c|c|}
\hline Star & \multicolumn{3}{|c|}{ a 1903.0} & $\begin{array}{l}\text { Red. to } \\
\text { app. place }\end{array}$ & \multicolumn{2}{|c|}{$\delta 1903.0$} & $\begin{array}{l}\text { Red. to } \\
\text { app. place }\end{array}$ & \multirow[b]{2}{*}{ Santini 2537} \\
\hline 1 & $22^{\mathrm{h}}$ & $27^{\mathrm{m}}$ & $14 \$ 43$ & $+2 s 61$ & $-11^{\circ} 17^{\prime}$ & $15^{\prime \prime} 1$ & +19.1 & \\
\hline 2 & 22 & 27 & 37.35 & +2.61 & $-11 \quad 18$ & 19.2 & +19.1 & Micrometer comparison with (1) \\
\hline 3 & 22 & 46 & 11.20 & +2.59 & -848 & 54.9 & +19.1 & BD. $-9.6073 . \quad 1$ obs. Vienna A.G. Zone 186. \\
\hline & \multicolumn{3}{|c|}{ a 1904.0} & & \multicolumn{2}{|c|}{$\delta 1904.0$} & & \\
\hline 4 & 23 & 15 & 17.26 & -0.58 & $-4 \quad 26$ & 30.7 & -1.1 & Cordoba Gen. Catal. 31629. (P.M. applied.) \\
\hline 5 & 23 & 21 & 44.33 & -0.49 & $-4 \quad 23$ & 20.4 & -1.9 & Micrometer comparison with (4). \\
\hline 6 & 23 & 34 & 27.92 & -0.47 & -241 & 10.8 & -6.4 & Weisse-Bessel II. $23 \div 655$ \\
\hline 7 & 23 & 35 & 34.33 & -0.47 & $-2 \quad 35$ & 03.9 & -6.4 & Micrometer comparison with (6). \\
\hline
\end{tabular}

These observations conclude the series published in Lick Observatory Bulletin Number 49. They were all made with the 36 -inch telescope. The comet, after fading in October, gained very perceptibly in brightness during December and was not very difficult to observe in January. Stormy weather prevented further observations.

R. G. Aitrken.

January $14,1905$. 